\title{
PERFORMANCE OF FOOD-TYPE SOYBEAN GENOTYPES AND THEIR POSSIBILITY FOR ADAPTATION TO BRAZILIAN LATITUDES ${ }^{1}$
}

\author{
EDSON PEREZ GUERRA ${ }^{2}$, DEONISIO DESTRO ${ }^{3}$, LILIAN AZEVEDO MIRANDA ${ }^{4}$ and RICARDO MONTALVÁN ${ }^{5}$
}

\begin{abstract}
This work was conducted at the Universidade Estadual de Londrina (UEL), in Londrina, State of Paraná, Brazil, with the goal to study food-type soybean (Glycine max (L.) Merrill) genotypes performance for use in cultivation or crosses. A total of 104 genotypes were analyzed: 88 were foodtype with large seeds, eight were food-type with small seeds, and eight-grain types adapted cultivars. The experimental plan was in randomized complete block design with four replications, and 12 traits of agronomic importance were considered. Genetic diversity was observed in the food-type germplasm. There were some genotypes with high yield adapted to a normal period of sowing. Soybean genetic improvement programs for direct human consumption in Brazil, either by means of Asiatic pure lines or by means of the incorporation of genes for late flowering in short-day conditions in this lines is highly viable.
\end{abstract}

Index terms: agronomic characters, genetic breeding, germplasm, Glycine max.

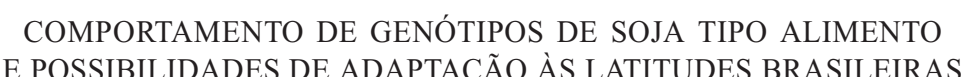

RESUMO - O presente trabalho foi desenvolvido na Universidade Estadual de Londrina (UEL), em Londrina, PR, com o objetivo de estudar o comportamento de genótipos de soja (Glycine max (L.) Merrill) tipo alimento, para posterior uso como cultivares ou em cruzamentos. Foram avaliados 104 genótipos, sendo 88 do tipo alimento, de sementes grandes, oito do tipo alimento, de sementes pequenas, e oito adaptados, tipo grão. $\mathrm{O}$ delineamento foi o de blocos completos ao acaso, com quatro repetições, considerando 12 caracteres de importância agronômica. Foi observada uma grande diversidade genética no germoplasma tipo alimento, havendo genótipos com alta produtividade e adaptados para época normal de semeadura. Programas de melhoramento genético da soja para consumo humano direto, no Brasil, são viáveis, seja pelo uso direto de linhagens asiáticas, seja por meio da incorporação de genes para florescimento tardio, em dias curtos, nestas linhagens.

Termos para indexação: caracteres agronômicos, melhoramento genético, germoplasma, Glycine max.

\section{INTRODUCTION}

Historical records suggest that soybean (Glycine $\max (\mathrm{L}$.$) Merrill) was domesticated in the Northeast$

\footnotetext{
${ }^{1}$ Accepted for publication on February 17, 1998.

Part of a thesis presented by the first author to Universidade Estadual de Londrina (UEL).

2 Eng. Agr., M.Sc., Prof. Assistente, Dep. de Agronomia, PUC-PR, Caixa Postal 16210, CEP 81611-970 Curitiba PR. E-mail: guerra@rla01.pucpr.br

${ }^{3}$ Eng. Agr., Dr., Prof. Adjunto, Dep. de Agronomia, UEL. E-mail: ddestro@uel.br

${ }^{4}$ Bióloga, Doutoranda em Ciência de Alimentos, UEL.

${ }^{5}$ Eng. Agr., D.Sc., Prof. Adjunto, Dep. de Agronomia, UNIOESTE, Caixa Postal 91, CEP 85960-000 Mal. Cândido Rondon, PR. Bolsista do CNPq.
}

of China, where its use is registered as one of the first food crops for humans (Silveira et al., 1989). After being introduced and improved in the United States, soybean was brought to Brazil where it has reached international recognition for high grain production since 1973 (Vello, 1992). Almost all grain type of soybean production is destined to foreign and domestic industries for oil and meal production. The soybean meal is almost entirely used for animal food and the oil is for human consumption.

There are several advantages, however, in the use of soybean for food: a) it is easily available for domestic consumption and can be cultivated anywhere in the country; b) it has the highest protein content among all the crop species grown in Brazil, and it also has a higher caloric power along with some other 
nutritional qualities; c) its protein supply cost is lower than that of the other conventional sources; d) it has a variety of cooking applications and can be part of most of traditional Brazilian cookery; and e) soybean has been used for millenniums in the Far East, and its human consumption increases in geometric progression in industrialized countries (Carrão-Panizzi, 1988).

Soybean directly used as food must have some especial characteristics, such as: a) a better quality and higher protein content; b) lower oil content and lower linolenic and linoleic unsaturated fatty acids and absence of lipoxigenases 1,2 and 3; c) sweet taste resembling nuts; d) light color seeds (cotyledons, tegument and hilum with similar colors, preferentially $\tan$ ), appropriate size to the kind of food; and e) fast cooking (Destro, 1991; Vello, 1992). Soybean for industry uses generally does not have all these characteristics, which makes it difficult to use as food supply. Eastern cultivars for human consumption have adaptation problems due to Brazilian photoperiodical conditions, resulting in lower productivity. Genotypes developed in Asia (with a latitude of approximately $40^{\circ}$ ) have early flowering when cultivated under short-day conditions. In Brazil, earlier flowering is associated with determinate stem growth habit, which reduces the number of internodes, plant height, and yield (Santos, 1988).

Carrão-Panizzi \& Meira (1989) made the characterization and analysis of 83 genotypes on food-type soybean germplasm collection from Embrapa-CNPSo and suggested nine quantitative traits of agronomic importance and nine qualitative traits for genotype identification and classification.

However, information about performance on foodtype soybean germplasm with breeding objectives are scarce in the literature.

The objective of this work was to study the performance of soybean genotypes appropriate to human consumption, in field conditions, for selection of the best genotypes for use as high yielding cultivars or in crosses.

\section{MATERIAL AND METHODS}

This experiment has utilized 104 soybean genotypes, 88 of which are food-type soybean genotypes with large seeds of either vegetable or "Edamame" kind, eight are food-type soybean genotypes with small seeds of either natto or sprout kind, and eight soybean cultivars adapted to Brazilian cultivation conditions, which were used as control samples. "Davis Marrom" has brown seeds and is an isogenic line selected from Davis cultivar, which presents yellow seeds. As these genotypes differ apparently only in seed color, "Davis Marrom" was considered adapted to Brazilian cultivation conditions.

The experiment was conducted in Londrina in 1991, on a soil classified as "terra roxa estruturada eutrófica" (Hapludult). The experimental plan was a randomized complete block design with four replications. Each plot included one $3.0-\mathrm{m}$ row, with 12 plants each, with an average distance of $0.25 \mathrm{~m}$ between plants and of $0.90 \mathrm{~m}$ between plots. Insecticides were applied during the experiment to control the stink bug population.

The following qualitative traits were registered: FC: flower color (W: white and P: purple); PC: pubescence color (Gy: gray and Br: brown); CSC: color of seed coat (Y: yellow, Bl: black, Gn: green, and Br: brown); HC: hilum color (L: light, Bl: black, LB: light brown and DB: dark brown).

The quantitative traits assessed were as follows: NDF: number of days to the beginning of flowering was the period between sowing and the first flower anthesis $\left(R_{1}\right.$ stage of the scale of Fehr \& Caviness (1977)); PHF: plant height $(\mathrm{cm})$ at the beginning of flowering was measured as the distance between soil and the inflorescence insertion most distant from main stem, analyzed on $\mathrm{R}_{1}$ stage; NDM: number of days to maturity is the period between sowing and the day in which approximately $95 \%$ of the pods were mature $\left(\mathrm{R}_{8}\right.$ stage of the scale of Fehr \& Caviness (1977)); PHM: plant height $(\mathrm{cm})$ at maturity was measured as the distance between the soil and the inflorescence insertion most distant from main stem, analyzed on $\mathrm{R}_{8}$ stage; AW: agronomic worth was visually analyzed at maturity (the grade scale varied from 1 to 5 , in which 1 corresponded to a bad plant and 5 to an excellent plant) and represents a visual rate of the plant global merit for a series of adaptive traits such as: number of pods, plant strength and health, resistance to lodging, shattering resistance, and less leaf retention after maturity (Hiromoto, 1990); NIM: number of internodes during maturity was counted from the cotyledon node to the top of the main stem; IPY: individual plant yield was evaluated after pod threshing of individual plants (data were collected in grams per plant), and the average data of each plot was used for analysis; HSW: one hundred seed weight was calculated taking the weight of 100 seeds per individual plant from four random plants per plot, and the average of the four 
plants was used for analysis; RP: reproductive period was the number of days between the beginning of flowering and maturity $(\mathrm{RP}=\mathrm{NDM}-\mathrm{NDF}) ; \% \mathrm{RP}$ : reproductive period percentage was the ratio, in percentage, of the number of days between flowering and maturity and the number of days for maturity $(\% \mathrm{RP}=(\mathrm{RP} / \mathrm{NDM}) \times 100)$; $\mathrm{RG}$ : reproductive growth after flowering was plant growth $(\mathrm{cm})$ between the beginning of flowering and maturity $(\mathrm{RG}=\mathrm{PHM}-$ PHF); \%RG: reproductive growth percentage was the ratio, in percentage, of the plant growth after flowering, and its height after maturity $(\% \mathrm{RG}=(\mathrm{RG} / \mathrm{PHM}) \times 100)$.

Besides the univariated variance analysis, the average comparisons based on Scott \& Knott (1974) criteria were also done for the quantitative characteristics. Pacova (1992) suggested that such method is used to detect differences among average groups of treatments for balanced designs, especially those in which a great number of genotypes is compared. The method essentially consists of the ramified or hierarchical spelling process, in which $n$ treatment averages are divided in two large groups. Each group is further subdivided in two other groups and so on, in a continuous process of subdivision, so that each sub-group has a pattern of mutual similarity to the data set. The subdivision process ends when there is no significant difference between two groups. The analysis provides a maximum intra-cluster homogeneity and, consequently, a maximum inter-cluster heterogeneity. The method allows the separation of treatments in classes, which are represented by one letter for each analyzed variable. Because IPY is one of the main characteristics in parental selection for crosses, treatments with higher IPY (letter 'A') were chosen.

\section{RESULTS AND DISCUSSION}

The analysis of variance of the 12 traits of agronomic importance indicated significant differences $(\mathrm{P}<0.01)$ among genotypes for all analyzed characters (Table 1). The coefficient of variation (C.V.) of the characters were small, with exception of IPY (26.4\%), RG (26.2\%) and \%RG (26.6\%). Destro (1991), in a study of food-type soybean genotypes reported similar C.V. values for IPY and for number of seeds per plant.

The means of the evaluated characters and the Scott \& Knott significance test are presented in Table 2. Diversity among genotypes was evident for all traits, with emphasis for NDF (from 31.2 to 84.6 days), for NDM (from 96.3 to 174.3 days), and for PHM (from 19.7 to $157.9 \mathrm{~cm}$ ).

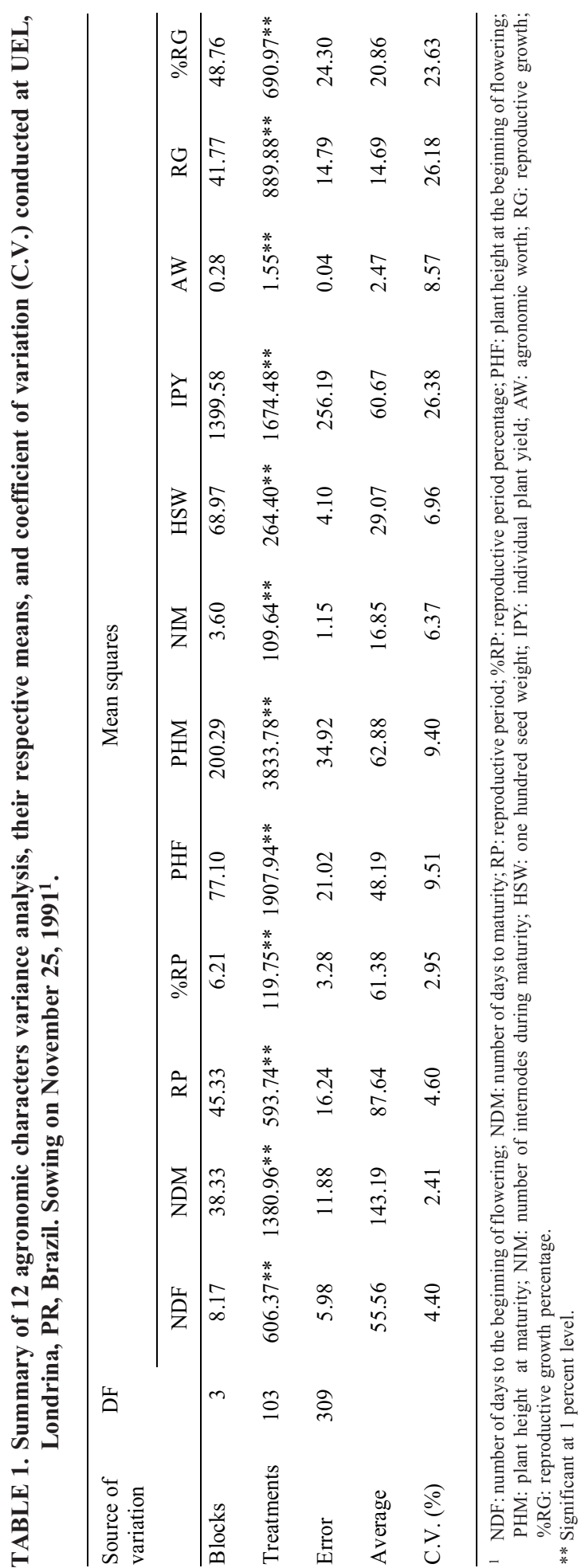

Pesq. agropec. bras., Brasília, v.34, n.4, p.575-583, abr. 1999 


\begin{tabular}{|c|c|c|c|c|c|c|c|c|c|c|c|c|c|c|c|c|}
\hline \multirow[t]{2}{*}{ Cultivar $^{2}$} & \multicolumn{12}{|c|}{ Quantitative characters ${ }^{3}$} & \multicolumn{4}{|c|}{ Qualitative characters } \\
\hline & NDF & NDM & $\mathrm{RP}$ & $\% \mathrm{RP}$ & PHF & PHM & NIM & HSW & IPY & AW & RG & $\%$ RG & FC & $\mathrm{PC}$ & $\mathrm{CSC}$ & $\mathrm{HC}$ \\
\hline BR-27 & $73.6 \mathrm{c}$ & $166.0 \mathrm{~b}$ & $92.4 \mathrm{~d}$ & $55.6 \mathrm{f}$ & $83.8 \mathrm{c}$ & $103.3 \mathrm{e}$ & $21.5 \mathrm{~d}$ & $22.5 \mathrm{e}$ & $68.6 \mathrm{~b}$ & $3.56 \mathrm{~b}$ & $19.5 \mathrm{e}$ & $19.0 \mathrm{~g}$ & W & $\mathrm{Br}$ & $\mathrm{Y}$ & $\mathrm{Bl}$ \\
\hline EMBRAPA-4 & $50.7 \mathrm{~h}$ & $137.3 \mathrm{~h}$ & $86.7 \mathrm{e}$ & $63.1 \mathrm{c}$ & $41.9 \mathrm{~h}$ & $54.4 \mathrm{i}$ & $15.3 \mathrm{~g}$ & $20.9 \mathrm{f}$ & $75.2 \mathrm{~b}$ & $2.36 \mathrm{e}$ & $12.5 \mathrm{f}$ & $22.9 \mathrm{f}$ & $P$ & Gy & $\mathrm{Y}$ & LB \\
\hline IAC-12 & $56.1 \mathrm{~g}$ & $140.5 \mathrm{~h}$ & $84.4 \mathrm{e}$ & $60.1 \mathrm{~d}$ & $49.9 \mathrm{~g}$ & $63.9 \mathrm{i}$ & $18.1 \mathrm{f}$ & $14.1 \mathrm{~g}$ & $81.1 \mathrm{a}$ & $2.86 \mathrm{~d}$ & $13.9 \mathrm{f}$ & $21.8 \mathrm{f}$ & W & $\mathrm{Br}$ & $\mathrm{Y}$ & LB \\
\hline IAC-100 & $58.9 \mathrm{f}$ & $133.0 \mathrm{i}$ & $74.1 \mathrm{~g}$ & $55.7 \mathrm{f}$ & $39.1 \mathrm{~h}$ & $58.9 \mathrm{i}$ & $17.4 \mathrm{f}$ & $11.8 \mathrm{~h}$ & $61.8 \mathrm{~b}$ & $2.58 \mathrm{e}$ & $19.8 \mathrm{e}$ & $33.4 \mathrm{e}$ & W & $\mathrm{Br}$ & $\mathrm{Y}$ & $\mathrm{L}$ \\
\hline Doko Preta & $84.6 \mathrm{a}$ & $162.6 \mathrm{c}$ & $78.0 \mathrm{f}$ & $48.0 \mathrm{~h}$ & $97.9 \mathrm{a}$ & $100.9 \mathrm{e}$ & $22.2 \mathrm{~d}$ & $16.0 \mathrm{~g}$ & $47.0 \mathrm{c}$ & $3.18 \mathrm{c}$ & $3.0 \mathrm{~h}$ & $2.9 \mathrm{i}$ & W & $\mathrm{Br}$ & $\mathrm{Bl}$ & $\mathrm{B} 1$ \\
\hline Davis Marrom & $53.1 \mathrm{~g}$ & $141.3 \mathrm{~g}$ & $88.2 \mathrm{~d}$ & $62.3 \mathrm{~d}$ & $38.3 \mathrm{~h}$ & $46.3 \mathrm{j}$ & $15.0 \mathrm{~g}$ & $19.1 \mathrm{f}$ & $91.0 \mathrm{a}$ & $2.46 \mathrm{e}$ & $8.0 \mathrm{~g}$ & $17.3 \mathrm{~g}$ & W & Gy & $\mathrm{Br}$ & DB \\
\hline Paraná Marrom & $49.0 \mathrm{i}$ & $119.7 \mathrm{k}$ & $70.7 \mathrm{~g}$ & $59.0 \mathrm{e}$ & $38.2 \mathrm{~h}$ & $47.9 \mathrm{j}$ & $14.2 \mathrm{~g}$ & $16.5 \mathrm{~g}$ & $60.4 \mathrm{~b}$ & $2.12 \mathrm{f}$ & $9.7 \mathrm{~g}$ & $20.3 \mathrm{f}$ & W & Gy & $\mathrm{Br}$ & DB \\
\hline IAC-4 Marrom & $70.4 \mathrm{~d}$ & $157.8 \mathrm{~d}$ & $87.4 \mathrm{~d}$ & $55.3 \mathrm{f}$ & $64.6 \mathrm{e}$ & $74.6 \mathrm{~h}$ & $20.3 \mathrm{e}$ & $14.7 \mathrm{~g}$ & $66.4 \mathrm{~b}$ & $2.84 \mathrm{~d}$ & $10.0 \mathrm{~g}$ & $13.3 \mathrm{~h}$ & W & Gy & $\mathrm{Br}$ & DB \\
\hline Delsta & $55.4 \mathrm{~g}$ & $146.4 \mathrm{f}$ & $91.0 \mathrm{~d}$ & $62.2 \mathrm{~d}$ & $38.4 \mathrm{~h}$ & $48.6 \mathrm{j}$ & $15.9 \mathrm{f}$ & $31.0 \mathrm{c}$ & $74.5 \mathrm{~b}$ & $2.33 \mathrm{f}$ & $10.2 \mathrm{~g}$ & $21.0 \mathrm{f}$ & $P$ & Gy & $\mathrm{Y}$ & LB \\
\hline Faz. Progresso & $77.1 \mathrm{~b}$ & $167.1 \mathrm{~b}$ & $90.0 \mathrm{~d}$ & $53.8 \mathrm{~g}$ & $75.3 \mathrm{~d}$ & $82.5 \mathrm{~g}$ & $23.7 \mathrm{~d}$ & $30.2 \mathrm{~d}$ & $99.5 \mathrm{a}$ & $3.51 \mathrm{~b}$ & $7.2 \mathrm{~g}$ & $8.7 \mathrm{~h}$ & $\mathrm{P}$ & Gy & $\mathrm{Y}$ & LB \\
\hline F80-3309 & $51.2 \mathrm{~h}$ & $150.2 \mathrm{f}$ & $99.0 \mathrm{~b}$ & $65.9 \mathrm{~b}$ & $38.3 \mathrm{~h}$ & $45.9 \mathrm{j}$ & $13.3 \mathrm{~h}$ & $24.1 \mathrm{e}$ & $49.5 \mathrm{c}$ & $2.39 \mathrm{e}$ & $7.6 \mathrm{~g}$ & $16.7 \mathrm{~g}$ & $P$ & $\mathrm{Br}$ & $\mathrm{Y}$ & DB \\
\hline F80-6717 & $64.2 \mathrm{e}$ & $168.3 \mathrm{~b}$ & $104.1 \mathrm{~b}$ & $61.9 \mathrm{~d}$ & $63.0 \mathrm{e}$ & $72.6 \mathrm{~h}$ & $16.2 \mathrm{f}$ & $32.1 \mathrm{c}$ & $77.7 \mathrm{~b}$ & $3.17 \mathrm{c}$ & $9.7 \mathrm{~g}$ & $13.3 \mathrm{~h}$ & $P$ & $\mathrm{Br}$ & $\mathrm{Bl}$ & $\mathrm{B} 1$ \\
\hline F80-6933 & $45.4 \mathrm{j}$ & $136.4 \mathrm{~h}$ & $91.0 \mathrm{~d}$ & $66.7 \mathrm{a}$ & $34.2 \mathrm{i}$ & $38.3 \mathrm{k}$ & $11.3 \mathrm{~h}$ & $36.2 \mathrm{~b}$ & $66.5 b$ & $2.07 \mathrm{f}$ & $4.1 \mathrm{~h}$ & $10.7 \mathrm{~h}$ & $P$ & $\mathrm{Br}$ & $\mathrm{Bl}$ & $\mathrm{Bl}$ \\
\hline F81-9136 & $50.8 \mathrm{~h}$ & $158.3 \mathrm{~d}$ & $107.5 \mathrm{a}$ & $67.9 \mathrm{a}$ & $37.0 \mathrm{~h}$ & $49.8 \mathrm{j}$ & $14.4 \mathrm{~g}$ & $34.1 \mathrm{c}$ & $70.4 \mathrm{~b}$ & $2.29 \mathrm{f}$ & $12.8 \mathrm{f}$ & $25.6 \mathrm{f}$ & $\mathrm{P}$ & $\mathrm{Br}$ & $\mathrm{Y}$ & $\mathrm{Bl}$ \\
\hline F82-5628 & $53.9 \mathrm{~g}$ & $154.9 \mathrm{e}$ & $101.0 \mathrm{~b}$ & $65.2 \mathrm{~b}$ & $42.7 \mathrm{~h}$ & $58.2 \mathrm{i}$ & $16.0 \mathrm{f}$ & $34.5 \mathrm{c}$ & $55.4 \mathrm{c}$ & $2.70 \mathrm{~d}$ & $15.5 \mathrm{f}$ & $26.3 \mathrm{f}$ & $\mathrm{P}$ & $\mathrm{Br}$ & $\mathrm{B} 1$ & $\mathrm{Bl}$ \\
\hline F82-5630 & $50.8 \mathrm{~h}$ & $157.7 \mathrm{~d}$ & $106.9 \mathrm{a}$ & $67.8 \mathrm{a}$ & $34.6 \mathrm{i}$ & $46.9 \mathrm{j}$ & $13.7 \mathrm{~h}$ & $35.8 \mathrm{~b}$ & $40.7 \mathrm{c}$ & $2.12 \mathrm{f}$ & $12.3 \mathrm{~g}$ & $26.4 \mathrm{f}$ & $\mathrm{P}$ & $\mathrm{Br}$ & $\mathrm{Bl}$ & $\mathrm{Bl}$ \\
\hline F82-5721 & $52.5 \mathrm{~h}$ & $153.5 \mathrm{e}$ & $101.0 \mathrm{~b}$ & $65.7 \mathrm{~b}$ & $41.4 \mathrm{~h}$ & $49.3 \mathrm{j}$ & $13.9 \mathrm{~g}$ & $34.3 \mathrm{c}$ & $83.8 \mathrm{a}$ & $2.48 \mathrm{e}$ & $7.9 \mathrm{~g}$ & $16.0 \mathrm{~g}$ & $\mathrm{P}$ & $\mathrm{Br}$ & Bl & $\mathrm{B} 1$ \\
\hline F82-5722A & $47.8 \mathrm{i}$ & $157.3 \mathrm{~d}$ & $109.5 \mathrm{a}$ & $69.4 \mathrm{a}$ & $46.0 \mathrm{~g}$ & $59.8 \mathrm{i}$ & $16.4 \mathrm{f}$ & $38.9 \mathrm{~b}$ & $88.5 \mathrm{a}$ & $2.83 \mathrm{~d}$ & $13.8 \mathrm{f}$ & $23.1 \mathrm{f}$ & $P$ & $\mathrm{Br}$ & Gn & $\mathrm{Bl}$ \\
\hline F82-5722P & $57.6 \mathrm{f}$ & $157.0 \mathrm{~d}$ & $99.4 \mathrm{~b}$ & $63.3 \mathrm{c}$ & $46.7 \mathrm{~g}$ & $60.1 \mathrm{i}$ & $16.0 \mathrm{f}$ & $42.0 \mathrm{a}$ & $90.9 \mathrm{a}$ & $2.91 \mathrm{~d}$ & $13.3 \mathrm{f}$ & $22.0 \mathrm{f}$ & $P$ & $\mathrm{Br}$ & $\mathrm{Bl}$ & $\mathrm{B} 1$ \\
\hline F82-5767 & $52.1 \mathrm{~h}$ & $154.1 \mathrm{e}$ & $102.0 \mathrm{~b}$ & $66.2 \mathrm{~b}$ & $40.0 \mathrm{~h}$ & $42.3 \mathrm{k}$ & $11.9 \mathrm{~h}$ & $42.8 \mathrm{a}$ & $71.2 \mathrm{~b}$ & $2.29 \mathrm{f}$ & $2.3 \mathrm{~h}$ & $5.3 \mathrm{i}$ & $\mathrm{P}$ & $\mathrm{Br}$ & $\mathrm{Bl}$ & $\mathrm{Bl}$ \\
\hline F82-5769 & $52.0 \mathrm{~h}$ & $152.1 \mathrm{e}$ & $100.1 \mathrm{~b}$ & $65.8 \mathrm{~b}$ & $40.7 \mathrm{~h}$ & $51.2 \mathrm{j}$ & $14.7 \mathrm{~g}$ & $36.9 b$ & $92.4 \mathrm{a}$ & $2.52 \mathrm{e}$ & $10.5 \mathrm{~g}$ & $20.4 \mathrm{f}$ & W & $\mathrm{Br}$ & $\mathrm{Bl}$ & $\mathrm{Bl}$ \\
\hline F82-5782 & $58.1 \mathrm{f}$ & $149.5 \mathrm{f}$ & $91.4 \mathrm{~d}$ & $61.2 \mathrm{~d}$ & $49.4 \mathrm{~g}$ & $64.1 \mathrm{i}$ & $17.1 \mathrm{f}$ & $38.3 b$ & $112.3 \mathrm{a}$ & $2.70 \mathrm{~d}$ & $14.7 \mathrm{f}$ & 23.0f & $P$ & $\mathrm{Br}$ & $\mathrm{B} 1$ & Bl \\
\hline F82-5783 & $48.8 \mathrm{i}$ & $152.8 \mathrm{e}$ & $104.0 \mathrm{~b}$ & $68.1 \mathrm{a}$ & $26.6 \mathrm{i}$ & 35.21 & $12.5 \mathrm{~h}$ & $36.5 \mathrm{~b}$ & $67.2 \mathrm{~b}$ & $1.98 \mathrm{~g}$ & $8.5 \mathrm{~g}$ & $22.1 \mathrm{f}$ & $\mathrm{P}$ & $\mathrm{Br}$ & $\mathrm{Bl}$ & $\mathrm{Bl}$ \\
\hline F82-5803 & $53.5 \mathrm{~g}$ & $152.3 \mathrm{e}$ & $98.8 \mathrm{~b}$ & $64.9 \mathrm{~b}$ & $39.3 \mathrm{~h}$ & $48.3 \mathrm{j}$ & $14.0 \mathrm{~g}$ & $33.2 \mathrm{c}$ & $91.0 \mathrm{a}$ & $2.56 \mathrm{e}$ & $9.0 \mathrm{~g}$ & $18.5 \mathrm{~g}$ & W & $\mathrm{Br}$ & $\mathrm{Y}$ & $\mathrm{Bl}$ \\
\hline F82-5812 & $50.3 \mathrm{~h}$ & $153.2 \mathrm{e}$ & $102.9 \mathrm{~b}$ & $67.2 \mathrm{a}$ & $34.2 \mathrm{i}$ & $40.9 \mathrm{k}$ & $12.5 \mathrm{~h}$ & $35.4 \mathrm{~b}$ & $67.3 b$ & $2.19 \mathrm{f}$ & $6.7 \mathrm{~g}$ & $15.8 \mathrm{~g}$ & W & $\mathrm{Br}$ & Gn & DB \\
\hline F82-5813 & $50.1 \mathrm{~h}$ & $145.3 \mathrm{~g}$ & $95.1 \mathrm{c}$ & $65.5 \mathrm{~b}$ & $33.3 \mathrm{i}$ & $41.7 \mathrm{k}$ & $14.2 \mathrm{~g}$ & $29.4 \mathrm{~d}$ & $90.0 \mathrm{a}$ & $2.40 \mathrm{e}$ & $8.3 \mathrm{~g}$ & $20.0 \mathrm{f}$ & W & $\mathrm{Br}$ & $\mathrm{Y}$ & DB \\
\hline F83-8202 & $62.2 \mathrm{e}$ & $165.0 \mathrm{~b}$ & $102.8 \mathrm{~b}$ & $62.3 \mathrm{~d}$ & $59.4 \mathrm{f}$ & $88.9 \mathrm{f}$ & $20.9 \mathrm{e}$ & $30.1 \mathrm{~d}$ & $79.6 \mathrm{~b}$ & $3.23 \mathrm{c}$ & $29.6 \mathrm{~d}$ & $33.2 \mathrm{e}$ & $\mathrm{P}$ & $\mathrm{Br}$ & $\mathrm{Bl}$ & $\mathrm{B} 1$ \\
\hline F83-7843 & $54.1 \mathrm{~g}$ & $151.1 \mathrm{e}$ & $97.0 \mathrm{~b}$ & $64.2 \mathrm{c}$ & $41.8 \mathrm{~h}$ & $50.4 \mathrm{j}$ & $15.4 \mathrm{~g}$ & $31.3 \mathrm{c}$ & $84.5 \mathrm{a}$ & $2.53 \mathrm{e}$ & $8.6 \mathrm{~g}$ & $16.9 \mathrm{~g}$ & $\mathrm{P}$ & Gy & $\mathrm{Y}$ & LB \\
\hline F83-7864 & $54.4 \mathrm{~g}$ & $157.5 \mathrm{~d}$ & $103.1 \mathrm{~b}$ & $65.4 \mathrm{~b}$ & $42.4 \mathrm{~h}$ & $50.0 \mathrm{j}$ & $15.0 \mathrm{~g}$ & $33.0 \mathrm{c}$ & $58.3 \mathrm{c}$ & $2.25 \mathrm{f}$ & $7.5 \mathrm{~g}$ & $15.1 \mathrm{~g}$ & W & $\mathrm{Br}$ & $\mathrm{Y}$ & Bl \\
\hline F83-7931 & $44.4 j$ & $136.5 \mathrm{~h}$ & $92.1 \mathrm{~d}$ & $67.5 \mathrm{a}$ & $28.8 \mathrm{i}$ & $34.8 \mathrm{~m}$ & $11.7 \mathrm{~h}$ & $24.7 \mathrm{e}$ & $30.5 \mathrm{~d}$ & $1.74 \mathrm{~g}$ & $6.0 \mathrm{~h}$ & $17.1 \mathrm{~g}$ & $\mathrm{P}$ & $\mathrm{Br}$ & $\mathrm{Y}$ & $\mathrm{B} 1$ \\
\hline F83-7959 & $55.1 \mathrm{~g}$ & $158.2 \mathrm{~d}$ & $103.0 \mathrm{~b}$ & $65.1 \mathrm{~b}$ & $36.2 \mathrm{~h}$ & $42.5 \mathrm{k}$ & $14.6 \mathrm{~g}$ & $31.4 \mathrm{c}$ & $39.8 \mathrm{c}$ & $2.03 \mathrm{~g}$ & $6.3 \mathrm{~h}$ & $14.8 \mathrm{~g}$ & $P$ & $\mathrm{Br}$ & $\mathrm{Y}$ & $\mathrm{Bl}$ \\
\hline F83-7977 & $53.1 \mathrm{~g}$ & $151.9 \mathrm{e}$ & $98.8 \mathrm{~b}$ & $65.0 \mathrm{~b}$ & $42.0 \mathrm{~h}$ & $49.7 \mathrm{j}$ & $14.6 \mathrm{~g}$ & $35.2 \mathrm{~b}$ & $67.9 b$ & $2.41 \mathrm{e}$ & $7.7 \mathrm{~g}$ & $15.4 \mathrm{~g}$ & W & Gy & $\mathrm{Y}$ & LB \\
\hline F83-7999 & $52.1 \mathrm{~h}$ & $151.2 \mathrm{e}$ & $99.1 \mathrm{~b}$ & $65.5 \mathrm{~b}$ & $38.3 \mathrm{~h}$ & $45.3 \mathrm{j}$ & $14.1 \mathrm{~g}$ & $34.1 \mathrm{c}$ & $64.0 \mathrm{~b}$ & $2.33 \mathrm{f}$ & $7.0 \mathrm{~g}$ & $15.5 \mathrm{~g}$ & $\mathrm{P}$ & $\mathrm{Br}$ & $\mathrm{Y}$ & $\mathrm{B} 1$ \\
\hline F83-8000 & $53.4 \mathrm{~g}$ & $161.5 \mathrm{c}$ & $108.2 \mathrm{a}$ & $66.9 \mathrm{a}$ & $36.8 \mathrm{~h}$ & $43.6 \mathrm{k}$ & $14.0 \mathrm{~g}$ & $33.5 \mathrm{c}$ & $53.8 \mathrm{c}$ & $2.21 \mathrm{f}$ & $6.9 \mathrm{~g}$ & $15.6 \mathrm{~g}$ & $\mathrm{P}$ & $\mathrm{Br}$ & $\mathrm{Y}$ & $\mathrm{B} 1$ \\
\hline F83-8012 & $70.3 \mathrm{~d}$ & $166.7 \mathrm{~b}$ & $96.4 \mathrm{c}$ & $57.8 \mathrm{e}$ & $78.6 \mathrm{c}$ & $96.6 \mathrm{e}$ & $23.6 \mathrm{~d}$ & $37.0 \mathrm{~b}$ & $103.5 \mathrm{a}$ & $3.71 \mathrm{a}$ & $18.0 \mathrm{f}$ & $18.5 \mathrm{~g}$ & $\mathrm{P}$ & $\mathrm{Br}$ & $\mathrm{B} 1$ & $\mathrm{Bl}$ \\
\hline F83-8017 & $55.9 \mathrm{~g}$ & $147.8 \mathrm{f}$ & $91.9 \mathrm{~d}$ & $62.2 \mathrm{~d}$ & $51.5 \mathrm{~g}$ & $65.7 \mathrm{~h}$ & $17.6 \mathrm{f}$ & $36.4 \mathrm{~b}$ & $83.0 \mathrm{a}$ & $2.89 \mathrm{~d}$ & $14.1 \mathrm{f}$ & $21.5 \mathrm{f}$ & $\mathrm{P}$ & $\mathrm{Br}$ & $\mathrm{Bl}$ & Bl \\
\hline
\end{tabular}


TABLE 2. Continuation.

\begin{tabular}{|c|c|c|c|c|c|c|c|c|c|c|c|c|c|c|c|c|}
\hline \multirow[t]{2}{*}{ Cultivar $^{2}$} & \multicolumn{12}{|c|}{ Quantitative characters ${ }^{3}$} & \multicolumn{4}{|c|}{ Qualitative characters } \\
\hline & NDF & NDM & $\mathrm{RP}$ & $\% \mathrm{RP}$ & PHF & PHM & NIM & HSW & IPY & $\mathrm{AW}$ & RG & $\% \mathrm{RG}$ & FC & $\mathrm{PC}$ & $\mathrm{CSC}$ & $\mathrm{HC}$ \\
\hline F83-8058P & $49.4 \mathrm{i}$ & $148.3 \mathrm{f}$ & $99.8 \mathrm{~b}$ & $67.2 \mathrm{a}$ & $34.0 \mathrm{i}$ & $39.4 \mathrm{k}$ & $13.2 \mathrm{~h}$ & $36.5 \mathrm{~b}$ & $76.0 \mathrm{~b}$ & $2.32 \mathrm{f}$ & $5.4 \mathrm{~h}$ & $14.2 \mathrm{~h}$ & $\mathrm{P}$ & $\mathrm{Br}$ & Bl & $\mathrm{Bl}$ \\
\hline F83-8117 & $78.4 \mathrm{~b}$ & $172.8 \mathrm{a}$ & $94.4 \mathrm{c}$ & $54.6 \mathrm{~g}$ & $83.7 \mathrm{c}$ & $89.2 \mathrm{f}$ & $19.1 \mathrm{e}$ & $42.3 \mathrm{a}$ & $69.8 \mathrm{~b}$ & $3.40 \mathrm{~b}$ & $5.4 \mathrm{~h}$ & $6.1 \mathrm{i}$ & $P$ & $\mathrm{Br}$ & B1 & B1 \\
\hline F83-8119 & $59.2 \mathrm{f}$ & $162.6 \mathrm{c}$ & $103.4 \mathrm{~b}$ & $63.6 \mathrm{c}$ & $44.7 \mathrm{~h}$ & $61.5 \mathrm{i}$ & $16.9 \mathrm{f}$ & $39.0 \mathrm{~b}$ & $79.0 \mathrm{~b}$ & $2.78 \mathrm{~d}$ & $16.8 \mathrm{f}$ & $27.3 \mathrm{f}$ & $\mathrm{P}$ & $\mathrm{Br}$ & Bl & $\mathrm{Bl}$ \\
\hline F83-8175 & $78.6 \mathrm{~b}$ & $174.3 \mathrm{a}$ & $95.8 \mathrm{c}$ & $54.9 \mathrm{f}$ & $84.9 \mathrm{c}$ & $93.9 \mathrm{f}$ & $20.4 \mathrm{e}$ & $36.8 \mathrm{~b}$ & $46.8 \mathrm{c}$ & $2.91 \mathrm{~d}$ & $9.1 \mathrm{~g}$ & $9.5 \mathrm{~h}$ & $\mathrm{P}$ & $\mathrm{Br}$ & $\mathrm{Bl}$ & $\mathrm{Bl}$ \\
\hline F83-8185 & $78.4 \mathrm{~b}$ & $170.3 \mathrm{a}$ & $92.2 \mathrm{~d}$ & $54.1 \mathrm{~g}$ & $80.8 \mathrm{c}$ & $84.0 \mathrm{~g}$ & $17.1 \mathrm{f}$ & $42.8 \mathrm{a}$ & $85.5 \mathrm{a}$ & $3.32 \mathrm{~b}$ & $3.2 \mathrm{~h}$ & $3.9 \mathrm{i}$ & $\mathrm{P}$ & $\mathrm{Br}$ & $\mathrm{Bl}$ & Bl \\
\hline F83-8192 & $79.5 \mathrm{~b}$ & $170.7 \mathrm{a}$ & $91.3 \mathrm{~d}$ & $53.4 \mathrm{~g}$ & $87.0 \mathrm{c}$ & $92.8 \mathrm{f}$ & $19.3 \mathrm{e}$ & $43.0 \mathrm{a}$ & $86.7 \mathrm{a}$ & $3.57 \mathrm{~b}$ & $5.8 \mathrm{~h}$ & $6.3 \mathrm{i}$ & $\mathrm{P}$ & $\mathrm{Br}$ & Bl & $\mathrm{Bl}$ \\
\hline F83-8203P & $47.2 \mathrm{i}$ & $145.3 \mathrm{~g}$ & $97.8 \mathrm{~b}$ & $67.4 a$ & $32.1 \mathrm{i}$ & $39.6 \mathrm{k}$ & $12.7 \mathrm{~h}$ & $31.5 \mathrm{c}$ & $56.6 \mathrm{c}$ & $2.02 \mathrm{~g}$ & $7.5 \mathrm{~g}$ & $18.6 \mathrm{~g}$ & $\mathrm{P}$ & $\mathrm{Br}$ & $\mathrm{B} 1$ & $\mathrm{~B} 1$ \\
\hline F83-8207AB & $53.0 \mathrm{~g}$ & $149.3 \mathrm{f}$ & $96.5 \mathrm{c}$ & $64.6 \mathrm{~b}$ & $39.5 \mathrm{~h}$ & $47.4 \mathrm{j}$ & $14.2 \mathrm{~g}$ & $35.9 \mathrm{~b}$ & $72.7 \mathrm{~b}$ & $2.42 \mathrm{e}$ & $7.9 \mathrm{~g}$ & $16.8 \mathrm{~g}$ & W & Gy & $\mathrm{Y}$ & LB \\
\hline F83-8211 & $50.2 \mathrm{~h}$ & $155.1 \mathrm{e}$ & $104.8 \mathrm{~b}$ & $67.6 \mathrm{a}$ & $36.8 \mathrm{~h}$ & $44.6 \mathrm{j}$ & $13.4 \mathrm{~h}$ & $31.5 \mathrm{c}$ & $72.5 b$ & $2.29 \mathrm{f}$ & $7.8 \mathrm{~g}$ & $17.5 \mathrm{~g}$ & $\mathrm{P}$ & $\mathrm{Br}$ & $\mathrm{B} 1$ & $\mathrm{Bl}$ \\
\hline F83-8240 & $54.8 \mathrm{~g}$ & $154.5 \mathrm{e}$ & $99.6 \mathrm{~b}$ & $64.5 \mathrm{c}$ & $49.0 \mathrm{~g}$ & $61.9 \mathrm{i}$ & $15.3 \mathrm{~g}$ & $32.3 \mathrm{c}$ & $70.9 \mathrm{~b}$ & $2.52 \mathrm{e}$ & $12.9 \mathrm{f}$ & $20.8 \mathrm{f}$ & $\mathrm{P}$ & $\mathrm{Br}$ & $\mathrm{B} 1$ & $\mathrm{Bl}$ \\
\hline F85-11346 & $57.0 \mathrm{f}$ & $169.5 \mathrm{a}$ & $112.5 \mathrm{a}$ & $66.4 \mathrm{~b}$ & $51.8 \mathrm{~g}$ & $65.2 \mathrm{~h}$ & $16.5 \mathrm{f}$ & $37.2 \mathrm{~b}$ & $63.3 \mathrm{~b}$ & $3.03 \mathrm{c}$ & $13.3 \mathrm{f}$ & $20.4 \mathrm{f}$ & W & $\mathrm{Br}$ & $\mathrm{Y}$ & $\mathrm{Bl}$ \\
\hline Kanrich & 31.81 & 105.31 & $73.5 \mathrm{~g}$ & $69.7 \mathrm{a}$ & $20.2 \mathrm{j}$ & $46.1 \mathrm{j}$ & $15.5 \mathrm{~g}$ & $31.7 \mathrm{c}$ & $29.4 \mathrm{~d}$ & $1.77 \mathrm{~g}$ & $25.9 \mathrm{~d}$ & $56.1 \mathrm{c}$ & $\mathrm{P}$ & $\mathrm{G}$ & Y & LB \\
\hline Kanro & 34.11 & $96.9 \mathrm{~m}$ & $62.7 \mathrm{~h}$ & $64.6 \mathrm{c}$ & $18.5 \mathrm{j}$ & $19.7 \mathrm{~m}$ & $8.4 \mathrm{j}$ & $27.7 \mathrm{~d}$ & $17.8 \mathrm{~d}$ & $1.23 \mathrm{~h}$ & $1.2 \mathrm{~h}$ & $5.9 \mathrm{i}$ & $\mathrm{P}$ & $\mathrm{Br}$ & $\mathrm{Y}$ & $\mathrm{L}$ \\
\hline L81-4590 & 31.21 & $96.3 \mathrm{~m}$ & $65.0 \mathrm{~h}$ & $67.5 \mathrm{a}$ & $17.4 j$ & $45.0 \mathrm{j}$ & $15.5 \mathrm{~g}$ & $19.9 \mathrm{f}$ & $42.4 \mathrm{c}$ & $1.97 \mathrm{~g}$ & $27.6 \mathrm{~d}$ & $61.4 \mathrm{~b}$ & W & $\mathrm{Br}$ & Y & $\mathrm{B} 1$ \\
\hline Late Giant & $45.0 \mathrm{j}$ & $146.7 \mathrm{f}$ & $101.7 \mathrm{~b}$ & $67.8 \mathrm{a}$ & $24.9 \mathrm{j}$ & 31.61 & $11.0 \mathrm{i}$ & $39.3 \mathrm{~b}$ & $53.1 \mathrm{c}$ & $1.95 \mathrm{~g}$ & $6.8 \mathrm{~g}$ & $21.5 \mathrm{f}$ & $\mathrm{P}$ & $\mathrm{Br}$ & $\mathrm{B} 1$ & $\mathrm{Bl}$ \\
\hline Mendota & 32.31 & $96.5 \mathrm{~m}$ & $64.2 \mathrm{~h}$ & $66.5 b$ & $14.6 \mathrm{j}$ & $26.1 \mathrm{~m}$ & $11.4 \mathrm{~h}$ & $22.5 \mathrm{e}$ & $24.7 \mathrm{~d}$ & $1.36 \mathrm{~h}$ & $11.4 \mathrm{~g}$ & $43.0 \mathrm{~d}$ & $\mathrm{P}$ & Gy & $\mathrm{Y}$ & $\mathrm{L}$ \\
\hline Nanda & $53.0 \mathrm{~g}$ & $140.0 \mathrm{~h}$ & $86.5 \mathrm{e}$ & $61.8 \mathrm{~d}$ & $38.2 \mathrm{~h}$ & $52.0 \mathrm{i}$ & $14.8 \mathrm{~g}$ & $23.1 \mathrm{e}$ & $36.1 \mathrm{~d}$ & $2.17 \mathrm{f}$ & $13.8 \mathrm{f}$ & $25.7 \mathrm{f}$ & $\mathrm{P}$ & Gy & $\mathrm{Y}$ & DB \\
\hline PI-133.226 & $72.6 \mathrm{c}$ & $150.8 \mathrm{e}$ & $78.2 \mathrm{f}$ & $51.8 \mathrm{~g}$ & $100.0 \mathrm{a}$ & $116.0 \mathrm{~d}$ & $22.2 \mathrm{~d}$ & $19.8 \mathrm{f}$ & $55.2 \mathrm{c}$ & $2.97 \mathrm{~d}$ & $16.1 \mathrm{f}$ & $13.9 \mathrm{~h}$ & W & Gy & $\mathrm{Y}$ & DB \\
\hline PI- 157.440 & $47.6 \mathrm{i}$ & $120.7 \mathrm{k}$ & $72.0 \mathrm{~g}$ & $59.6 \mathrm{~d}$ & $37.6 \mathrm{~h}$ & $40.3 \mathrm{k}$ & $12.1 \mathrm{~h}$ & $21.6 \mathrm{f}$ & $57.9 \mathrm{c}$ & $1.94 \mathrm{~g}$ & $2.7 \mathrm{~h}$ & $6.5 \mathrm{i}$ & W & Gy & $\mathrm{Y}$ & DB \\
\hline PI-423.909 & $59.8 \mathrm{f}$ & $153.8 \mathrm{e}$ & $94.1 \mathrm{c}$ & $61.2 \mathrm{~d}$ & $52.2 \mathrm{~g}$ & $109.2 \mathrm{~d}$ & $26.8 \mathrm{c}$ & $31.8 \mathrm{c}$ & $60.8 \mathrm{~b}$ & $3.52 \mathrm{~b}$ & $57.0 \mathrm{~b}$ & $52.2 \mathrm{c}$ & $\mathrm{P}$ & $\mathrm{Br}$ & $\mathrm{Y}$ & DB \\
\hline Soja Feira 86-13 & $47.8 \mathrm{i}$ & $138.0 \mathrm{~h}$ & $90.2 \mathrm{~d}$ & $65.3 \mathrm{~b}$ & $30.8 \mathrm{i}$ & $85.4 \mathrm{~g}$ & $20.5 \mathrm{e}$ & $37.8 \mathrm{~b}$ & $70.5 b$ & $2.44 \mathrm{e}$ & $54.7 \mathrm{~b}$ & $64.0 \mathrm{~b}$ & $\mathrm{P}$ & Gy & $\mathrm{Y}$ & DB \\
\hline Soja Feira 86-14 & $72.5 \mathrm{c}$ & $155.5 \mathrm{~d}$ & $83.0 \mathrm{e}$ & $53.4 \mathrm{~g}$ & $80.8 \mathrm{c}$ & $86.4 \mathrm{~g}$ & $22.3 \mathrm{~d}$ & $28.1 \mathrm{~d}$ & $61.7 \mathrm{~b}$ & $3.11 \mathrm{c}$ & $5.7 \mathrm{~h}$ & $6.5 \mathrm{i}$ & $P$ & Gy & Y & DB \\
\hline Aliança Preta & $69.8 \mathrm{~d}$ & $161.0 \mathrm{c}$ & $93.0 \mathrm{~d}$ & $57.8 \mathrm{e}$ & $79.6 \mathrm{c}$ & $98.7 \mathrm{e}$ & $21.3 \mathrm{~d}$ & $32.7 \mathrm{c}$ & $37.9 \mathrm{~d}$ & $3.17 \mathrm{c}$ & $19.2 \mathrm{e}$ & $19.4 \mathrm{~g}$ & $\mathrm{P}$ & $\mathrm{Br}$ & $\mathrm{Bl}$ & $\mathrm{Bl}$ \\
\hline Araçatuba & $73.7 \mathrm{c}$ & $153.3 \mathrm{e}$ & $79.6 \mathrm{f}$ & $51.9 \mathrm{~g}$ & $81.6 \mathrm{c}$ & $137.1 \mathrm{~b}$ & $28.8 \mathrm{~b}$ & $23.9 \mathrm{e}$ & $62.7 \mathrm{~b}$ & $3.62 \mathrm{a}$ & $55.5 \mathrm{~b}$ & $40.5 \mathrm{~d}$ & $\mathrm{P}$ & $\mathrm{Br}$ & $\mathrm{Y}$ & DB \\
\hline Biloxi 252n & $73.5 \mathrm{c}$ & $150.0 \mathrm{f}$ & $76.5 \mathrm{f}$ & $51.0 \mathrm{~h}$ & $73.6 \mathrm{~d}$ & $126.1 \mathrm{c}$ & $30.4 \mathrm{~b}$ & $21.4 \mathrm{f}$ & $47.5 \mathrm{c}$ & $3.22 \mathrm{c}$ & $52.5 \mathrm{~b}$ & $41.5 \mathrm{~d}$ & $\mathrm{P}$ & $\mathrm{Br}$ & $\mathrm{Br}$ & DB \\
\hline Cherokee & $69.3 \mathrm{~d}$ & $151.9 \mathrm{e}$ & $82.6 \mathrm{e}$ & $54.3 \mathrm{~g}$ & $57.7 \mathrm{f}$ & $82.1 \mathrm{~g}$ & $22.6 \mathrm{~d}$ & $29.1 \mathrm{~d}$ & $49.0 \mathrm{c}$ & $2.90 \mathrm{~d}$ & $24.4 \mathrm{~d}$ & $29.7 \mathrm{e}$ & $\mathrm{P}$ & Gy & Gn & DB \\
\hline FC 31-665 & $44.6 \mathrm{j}$ & $117.3 \mathrm{k}$ & $72.7 \mathrm{~g}$ & $62.0 \mathrm{~d}$ & $29.0 \mathrm{i}$ & 35.01 & $13.1 \mathrm{~h}$ & $31.9 \mathrm{c}$ & $58.4 \mathrm{c}$ & $1.88 \mathrm{~g}$ & $5.9 \mathrm{~h}$ & $17.0 \mathrm{~g}$ & $\mathrm{P}$ & Gy & $\mathrm{Y}$ & DB \\
\hline Hampton & $48.9 \mathrm{i}$ & $143.7 \mathrm{~g}$ & $94.7 \mathrm{c}$ & $65.9 \mathrm{~b}$ & $37.5 \mathrm{~h}$ & $39.2 \mathrm{k}$ & $13.9 \mathrm{~g}$ & $31.0 \mathrm{c}$ & $60.0 \mathrm{~b}$ & $2.03 \mathrm{~g}$ & $1.7 \mathrm{~h}$ & $4.3 \mathrm{i}$ & $P$ & Gy & $\mathrm{Y}$ & DB \\
\hline Hogyoku & $53.4 \mathrm{~g}$ & $153.7 \mathrm{e}$ & $100.3 \mathrm{~b}$ & $65.2 \mathrm{~b}$ & $39.6 \mathrm{~h}$ & $47.1 \mathrm{j}$ & $16.2 \mathrm{f}$ & $25.5 \mathrm{e}$ & $36.5 \mathrm{~d}$ & $2.08 \mathrm{f}$ & $7.5 \mathrm{~g}$ & $15.8 \mathrm{~g}$ & W & Gy & $\mathrm{Y}$ & DB \\
\hline Ivai & $53.6 \mathrm{~g}$ & $141.6 \mathrm{~g}$ & $88.0 \mathrm{~d}$ & $62.1 \mathrm{~d}$ & $47.3 \mathrm{~g}$ & $60.8 \mathrm{i}$ & $17.0 \mathrm{f}$ & $24.4 \mathrm{e}$ & $74.6 \mathrm{~b}$ & $2.54 \mathrm{e}$ & $13.5 \mathrm{f}$ & $22.1 \mathrm{f}$ & $\mathrm{P}$ & Gy & $\mathrm{Y}$ & LB \\
\hline Japão-1 & $38.2 \mathrm{k}$ & 105.71 & $67.5 \mathrm{~h}$ & $63.8 \mathrm{c}$ & $21.3 \mathrm{j}$ & $24.3 \mathrm{~m}$ & $10.1 \mathrm{i}$ & $33.5 \mathrm{c}$ & $24.4 \mathrm{~d}$ & $1.37 \mathrm{~h}$ & $3.0 \mathrm{~h}$ & $12.2 \mathrm{~h}$ & $\mathrm{~W}$ & $\mathrm{Br}$ & Gn & $\mathrm{Bl}$ \\
\hline Japão-2 & $43.9 \mathrm{j}$ & $124.0 \mathrm{j}$ & $80.1 \mathrm{f}$ & $64.5 \mathrm{c}$ & $31.0 \mathrm{i}$ & $38.2 \mathrm{k}$ & $12.1 \mathrm{~h}$ & $36.1 \mathrm{~b}$ & $47.1 \mathrm{c}$ & $1.77 \mathrm{~g}$ & $7.2 \mathrm{~g}$ & $17.5 \mathrm{~g}$ & $P$ & $\mathrm{Br}$ & Gn & $\mathrm{Bl}$ \\
\hline KS\#3xAkiyoshif-F7-2 & $52.2 \mathrm{~h}$ & $124.3 \mathrm{j}$ & $72.1 \mathrm{~g}$ & $58.0 \mathrm{e}$ & $38.6 \mathrm{~h}$ & $43.5 \mathrm{k}$ & $13.6 \mathrm{~h}$ & $27.7 \mathrm{~d}$ & $42.2 \mathrm{c}$ & $1.83 \mathrm{~g}$ & $4.8 \mathrm{~h}$ & $11.1 \mathrm{~h}$ & $\mathrm{P}$ & $\mathrm{Br}$ & $\mathrm{Y}$ & DB \\
\hline KS473 x SJ2-F7 & $57.0 \mathrm{f}$ & $135.4 \mathrm{~h}$ & $78.4 \mathrm{f}$ & $57.8 \mathrm{e}$ & $48.3 \mathrm{~g}$ & $59.4 \mathrm{i}$ & $16.6 \mathrm{f}$ & $24.1 \mathrm{e}$ & $45.4 \mathrm{c}$ & $2.33 \mathrm{f}$ & $11.1 \mathrm{~g}$ & $18.5 \mathrm{~g}$ & $P$ & $\mathrm{Br}$ & $\mathrm{Y}$ & DB \\
\hline Kurakake & $38.0 \mathrm{k}$ & 106.01 & $68.0 \mathrm{~h}$ & $64.1 \mathrm{c}$ & $23.3 \mathrm{j}$ & $24.9 \mathrm{~m}$ & $10.7 \mathrm{i}$ & $32.4 \mathrm{c}$ & $23.1 \mathrm{~d}$ & $1.38 \mathrm{~h}$ & $1.5 \mathrm{~h}$ & $6.3 \mathrm{i}$ & $\mathrm{W}$ & $\mathrm{Br}$ & Gn & $\mathrm{Bl}$ \\
\hline Majós & $51.9 \mathrm{~h}$ & $145.0 \mathrm{~g}$ & $93.0 \mathrm{~d}$ & $64.2 \mathrm{c}$ & $40.1 \mathrm{~h}$ & $45.3 \mathrm{j}$ & $15.3 \mathrm{~g}$ & $35.8 \mathrm{~b}$ & $97.3 \mathrm{a}$ & $2.46 \mathrm{e}$ & $5.2 \mathrm{~h}$ & $11.5 \mathrm{~h}$ & $\mathrm{~W}$ & Gy & $\mathrm{Y}$ & LB \\
\hline Mammoth Yellow & $48.3 \mathrm{i}$ & $117.8 \mathrm{k}$ & $69.2 \mathrm{~g}$ & $59.0 \mathrm{e}$ & $35.7 \mathrm{~h}$ & $38.1 \mathrm{k}$ & $12.8 \mathrm{~h}$ & $28.2 \mathrm{~d}$ & $41.9 \mathrm{c}$ & $1.75 \mathrm{~g}$ & $2.3 \mathrm{~h}$ & $6.2 \mathrm{i}$ & $\mathrm{W}$ & Gy & $\mathrm{Y}$ & DB \\
\hline
\end{tabular}




\begin{tabular}{|c|c|c|c|c|c|c|c|c|c|c|c|c|c|c|c|c|}
\hline \multirow[t]{2}{*}{ Cultivar $^{2}$} & \multicolumn{12}{|c|}{ Quantitative characters ${ }^{3}$} & \multicolumn{4}{|c|}{ Qualitative characters } \\
\hline & NDF & NDM & RP & $\% \mathrm{RP}$ & PHF & PHM & NIM & HSW & IPY & AW & RG & $\% \mathrm{RG}$ & FC & $\mathrm{PC}$ & $\mathrm{CSC}$ & $\mathrm{HC}$ \\
\hline Miyashipoken & $45.2 \mathrm{j}$ & $121.7 \mathrm{j}$ & $76.6 \mathrm{f}$ & $62.9 \mathrm{c}$ & $32.7 \mathrm{i}$ & $38.8 \mathrm{k}$ & $12.3 \mathrm{~h}$ & $32.5 \mathrm{c}$ & $43.3 \mathrm{c}$ & $1.83 \mathrm{~g}$ & $6.2 \mathrm{~h}$ & $15.8 \mathrm{~g}$ & $\mathrm{P}$ & $\mathrm{Br}$ & Gn & $\mathrm{Bl}$ \\
\hline Nimame & 34.41 & 109.11 & $74.7 \mathrm{~g}$ & $68.4 \mathrm{a}$ & $28.5 \mathrm{i}$ & 32.51 & $10.3 \mathrm{i}$ & $34.5 \mathrm{c}$ & $28.5 \mathrm{~d}$ & $1.47 \mathrm{~h}$ & $4.0 \mathrm{~h}$ & $11.6 \mathrm{~h}$ & $\mathrm{P}$ & $\mathrm{Br}$ & $\mathrm{Y}$ & LB \\
\hline PI-80.441 & 34.41 & 105.41 & $70.9 \mathrm{~g}$ & $67.1 \mathrm{a}$ & $19.3 \mathrm{j}$ & $20.0 \mathrm{~m}$ & $8.9 \mathrm{j}$ & $29.6 \mathrm{~d}$ & $13.4 \mathrm{~d}$ & $1.07 \mathrm{~h}$ & $0.8 \mathrm{~h}$ & $3.7 \mathrm{i}$ & W & Gy & $\mathrm{Y}$ & LB \\
\hline PI-91.725-3 & $57.8 \mathrm{f}$ & $138.9 \mathrm{~h}$ & $81.0 \mathrm{f}$ & $58.2 \mathrm{e}$ & $48.4 \mathrm{~g}$ & $59.2 \mathrm{i}$ & $16.8 \mathrm{f}$ & $26.4 \mathrm{e}$ & $32.0 \mathrm{~d}$ & $2.38 \mathrm{e}$ & $10.8 \mathrm{~g}$ & $18.4 \mathrm{~g}$ & $P$ & $\mathrm{Br}$ & $\mathrm{Y}$ & DB \\
\hline PI-165.672 & $49.6 \mathrm{i}$ & $118.9 \mathrm{k}$ & $69.4 \mathrm{~g}$ & $58.3 \mathrm{e}$ & $45.6 \mathrm{~g}$ & $66.8 \mathrm{~h}$ & $16.0 \mathrm{f}$ & $33.1 \mathrm{c}$ & $55.2 \mathrm{c}$ & $2.51 \mathrm{e}$ & $21.2 \mathrm{e}$ & $31.5 \mathrm{e}$ & $\mathrm{P}$ & $\mathrm{Br}$ & Gn & $\mathrm{Bl}$ \\
\hline PI-165.676 & $53.8 \mathrm{~g}$ & $144.5 \mathrm{~g}$ & $90.6 \mathrm{~d}$ & $62.7 \mathrm{~d}$ & $39.6 \mathrm{~h}$ & $59.2 \mathrm{i}$ & $16.7 \mathrm{f}$ & $33.0 \mathrm{c}$ & $44.7 \mathrm{c}$ & $2.37 \mathrm{e}$ & $19.6 \mathrm{e}$ & $33.0 \mathrm{e}$ & $\mathrm{P}$ & $\mathrm{Br}$ & $\mathrm{Br}$ & DB \\
\hline PI-229.320 & $46.9 \mathrm{i}$ & $118.9 \mathrm{k}$ & $71.9 \mathrm{~g}$ & $60.5 \mathrm{~d}$ & $33.1 \mathrm{i}$ & 36.61 & $11.8 \mathrm{~h}$ & $26.8 \mathrm{e}$ & $50.1 \mathrm{c}$ & $1.76 \mathrm{~g}$ & $3.5 \mathrm{~h}$ & $9.5 \mathrm{~h}$ & $\mathrm{P}$ & $\mathrm{Br}$ & $\mathrm{Y}$ & DB \\
\hline PI-230.977 & $47.0 \mathrm{i}$ & $131.1 \mathrm{i}$ & $84.1 \mathrm{e}$ & $64.1 \mathrm{c}$ & $26.8 \mathrm{i}$ & 30.11 & $12.3 \mathrm{~h}$ & $40.1 \mathrm{a}$ & $68.0 \mathrm{~b}$ & $1.96 \mathrm{~g}$ & $3.2 \mathrm{~h}$ & $10.6 \mathrm{~h}$ & $\mathrm{P}$ & $\mathrm{Br}$ & Bl & $\mathrm{B} 1$ \\
\hline PI-230.977/S & $53.3 \mathrm{~g}$ & $145.8 \mathrm{~g}$ & $92.5 \mathrm{~d}$ & $63.5 \mathrm{c}$ & $43.1 \mathrm{~h}$ & $68.2 \mathrm{~h}$ & $17.7 \mathrm{f}$ & $36.5 \mathrm{~b}$ & $45.0 \mathrm{c}$ & $2.52 \mathrm{e}$ & $25.1 \mathrm{~d}$ & $36.6 \mathrm{~d}$ & $P$ & $\mathrm{Br}$ & $\mathrm{Y}$ & $\mathrm{B} 1$ \\
\hline PI-243.514 & $42.9 \mathrm{j}$ & $120.3 \mathrm{k}$ & $77.3 \mathrm{f}$ & $64.3 \mathrm{c}$ & $24.9 \mathrm{j}$ & $28.2 \mathrm{~m}$ & $11.8 \mathrm{~h}$ & $31.2 \mathrm{c}$ & $56.3 \mathrm{c}$ & $1.62 \mathrm{~g}$ & $3.3 \mathrm{~h}$ & $11.3 \mathrm{~h}$ & W & $\mathrm{Br}$ & $\mathrm{Y}$ & DB \\
\hline PL-1 & $70.6 \mathrm{~d}$ & $150.3 \mathrm{e}$ & $79.7 \mathrm{f}$ & $53.0 \mathrm{~g}$ & $75.4 \mathrm{~d}$ & $87.6 \mathrm{~g}$ & $21.4 \mathrm{~d}$ & $29.3 \mathrm{~d}$ & $68.5 b$ & $3.05 \mathrm{c}$ & $12.2 \mathrm{~g}$ & $14.0 \mathrm{~h}$ & $\mathrm{P}$ & $\mathrm{Br}$ & Y & DB \\
\hline Pluto & $44.8 \mathrm{j}$ & $117.7 \mathrm{k}$ & $73.0 \mathrm{~g}$ & $62.0 \mathrm{~d}$ & $35.5 \mathrm{~h}$ & $40.9 \mathrm{k}$ & $12.8 \mathrm{~h}$ & $23.4 \mathrm{e}$ & $37.8 \mathrm{~d}$ & $1.80 \mathrm{~g}$ & $5.5 \mathrm{~h}$ & $13.3 \mathrm{~h}$ & W & $\mathrm{Br}$ & $\mathrm{B} 1$ & $\mathrm{~B} 1$ \\
\hline Stwart & $63.0 \mathrm{e}$ & $152.7 \mathrm{e}$ & $89.7 \mathrm{~d}$ & $58.7 \mathrm{e}$ & $71.2 \mathrm{~d}$ & $93.9 \mathrm{f}$ & $22.2 \mathrm{~d}$ & $19.7 \mathrm{f}$ & $85.7 \mathrm{a}$ & $3.49 \mathrm{~b}$ & $22.7 \mathrm{e}$ & $24.1 \mathrm{f}$ & $\mathrm{W}$ & Gy & $\mathrm{Y}$ & DB \\
\hline Tadacha & $41.7 \mathrm{j}$ & 112.01 & $70.3 \mathrm{~g}$ & $62.6 \mathrm{~d}$ & $25.0 \mathrm{j}$ & $27.6 \mathrm{~m}$ & $10.4 \mathrm{i}$ & $26.6 \mathrm{e}$ & $18.6 \mathrm{~d}$ & $1.39 \mathrm{~h}$ & $2.6 \mathrm{~h}$ & $9.6 \mathrm{~h}$ & $\mathrm{~W}$ & $\mathrm{Br}$ & $\mathrm{Br}$ & DB \\
\hline Tamba & $56.5 \mathrm{f}$ & $153.2 \mathrm{e}$ & $96.7 \mathrm{~b}$ & $63.1 \mathrm{c}$ & $50.9 \mathrm{~g}$ & $75.1 \mathrm{~h}$ & $20.2 \mathrm{e}$ & $27.3 \mathrm{~d}$ & $51.5 \mathrm{c}$ & $2.58 \mathrm{e}$ & $24.2 \mathrm{~d}$ & $31.9 \mathrm{e}$ & $\mathrm{P}$ & $\mathrm{Br}$ & $\mathrm{B} 1$ & $\mathrm{Bl}$ \\
\hline Tarheel Black & $47.4 \mathrm{i}$ & $130.9 \mathrm{i}$ & $83.5 \mathrm{e}$ & $63.8 \mathrm{c}$ & $37.7 \mathrm{~h}$ & $41.8 \mathrm{k}$ & $13.1 \mathrm{~h}$ & $36.7 \mathrm{~b}$ & $57.4 \mathrm{c}$ & $2.06 \mathrm{f}$ & $4.0 \mathrm{~h}$ & $9.6 \mathrm{~h}$ & $\mathrm{P}$ & $\mathrm{Br}$ & $\mathrm{Bl}$ & $\mathrm{B} 1$ \\
\hline TMV & $72.5 \mathrm{c}$ & $153.1 \mathrm{e}$ & $80.6 \mathrm{f}$ & $52.6 \mathrm{~g}$ & $85.4 \mathrm{c}$ & $91.8 \mathrm{f}$ & $21.8 \mathrm{~d}$ & $25.6 \mathrm{e}$ & $100.3 \mathrm{a}$ & $3.41 \mathrm{~b}$ & $6.4 \mathrm{~h}$ & $6.9 \mathrm{i}$ & $\mathrm{P}$ & $\mathrm{Br}$ & $\mathrm{Y}$ & DB \\
\hline TK\#5 x Unknown-F7 & $49.6 \mathrm{i}$ & $128.7 \mathrm{i}$ & $79.1 \mathrm{f}$ & $61.4 \mathrm{~d}$ & $42.4 \mathrm{~h}$ & $64.8 \mathrm{~h}$ & $16.6 \mathrm{f}$ & $34.2 \mathrm{c}$ & $42.4 \mathrm{c}$ & $2.46 \mathrm{e}$ & $22.5 \mathrm{e}$ & $34.6 \mathrm{e}$ & $\mathrm{P}$ & $\mathrm{Br}$ & Gn & $\mathrm{Bl}$ \\
\hline TN\#4xPI-230970 F7-1 & $61.0 \mathrm{f}$ & $153.7 \mathrm{e}$ & $92.6 \mathrm{~d}$ & $60.3 \mathrm{~d}$ & $68.3 \mathrm{e}$ & $81.8 \mathrm{~g}$ & $18.0 \mathrm{f}$ & $23.9 \mathrm{e}$ & $46.2 \mathrm{c}$ & $2.60 \mathrm{e}$ & $13.5 \mathrm{f}$ & $16.4 \mathrm{~g}$ & $\mathrm{P}$ & $\mathrm{Br}$ & $\mathrm{Y}$ & DB \\
\hline TN\#4xPI-230970 F7-4 & $54.4 \mathrm{~g}$ & $139.9 \mathrm{~h}$ & $85.6 \mathrm{e}$ & $61.1 \mathrm{~d}$ & $45.3 \mathrm{~g}$ & $55.7 \mathrm{i}$ & $15.4 \mathrm{~g}$ & $28.1 \mathrm{~d}$ & $35.4 \mathrm{~d}$ & $2.00 \mathrm{~g}$ & $10.4 \mathrm{~g}$ & $18.7 \mathrm{~g}$ & $\mathrm{P}$ & $\mathrm{Br}$ & $\mathrm{Y}$ & DB \\
\hline Yamagataken & $44.1 \mathrm{j}$ & $123.6 \mathrm{j}$ & $79.5 \mathrm{f}$ & $64.3 \mathrm{c}$ & $29.7 \mathrm{i}$ & 33.81 & $12.0 \mathrm{~h}$ & $31.9 \mathrm{c}$ & $61.3 b$ & $1.85 \mathrm{~g}$ & $4.1 \mathrm{~h}$ & $12.2 \mathrm{~h}$ & $\mathrm{P}$ & Gy & Gn & $\mathrm{B} 1$ \\
\hline Wolverine & $44.0 \mathrm{j}$ & $116.8 \mathrm{k}$ & $72.8 \mathrm{~g}$ & $62.3 \mathrm{~d}$ & $26.5 \mathrm{i}$ & 31.51 & $11.9 \mathrm{~h}$ & $23.8 \mathrm{e}$ & $52.8 \mathrm{c}$ & $1.72 \mathrm{~g}$ & $5.0 \mathrm{~h}$ & $15.7 \mathrm{~g}$ & $P$ & $\mathrm{Br}$ & $\mathrm{Y}$ & $\mathrm{B} 1$ \\
\hline $64.64 \times \mathrm{KS} 473$ & $52.2 \mathrm{~h}$ & $138.8 \mathrm{~h}$ & $86.6 \mathrm{e}$ & $62.4 \mathrm{~d}$ & $49.9 \mathrm{~g}$ & $59.6 \mathrm{i}$ & $15.6 \mathrm{~g}$ & $23.6 \mathrm{e}$ & $56.3 \mathrm{c}$ & $2.22 \mathrm{f}$ & $9.7 \mathrm{~g}$ & $16.1 \mathrm{~g}$ & $\mathrm{P}$ & $\mathrm{Br}$ & $\mathrm{Y}$ & DB \\
\hline Easycook & $40.3 \mathrm{j}$ & $126.6 \mathrm{i}$ & $86.4 \mathrm{e}$ & $68.2 \mathrm{a}$ & $23.0 \mathrm{j}$ & $90.6 \mathrm{f}$ & $22.1 \mathrm{~d}$ & $18.4 \mathrm{f}$ & $69.3 \mathrm{~b}$ & $2.23 \mathrm{f}$ & $67.6 \mathrm{a}$ & $74.5 \mathrm{a}$ & $\mathrm{P}$ & Gy & $\mathrm{Y}$ & DB \\
\hline Easycook Marrom 4 & $73.5 \mathrm{c}$ & $148.0 \mathrm{f}$ & $74.5 \mathrm{~g}$ & $50.3 \mathrm{~h}$ & $90.6 \mathrm{~b}$ & $147.1 \mathrm{a}$ & $29.4 \mathrm{~b}$ & $15.7 \mathrm{~g}$ & $65.0 \mathrm{~b}$ & $2.99 \mathrm{~d}$ & $56.5 \mathrm{~b}$ & $38.1 \mathrm{~d}$ & $\mathrm{P}$ & $\mathrm{Br}$ & $\mathrm{B} 1$ & B1 \\
\hline Easycook Marrom 4-a & $69.2 \mathrm{~d}$ & $148.8 \mathrm{f}$ & $79.6 \mathrm{f}$ & $53.5 \mathrm{~g}$ & $85.0 \mathrm{c}$ & $149.7 \mathrm{a}$ & $28.2 \mathrm{c}$ & $17.9 \mathrm{~g}$ & $71.0 \mathrm{~b}$ & $3.22 \mathrm{c}$ & $64.7 \mathrm{a}$ & $43.2 \mathrm{~d}$ & $P$ & $\mathrm{Br}$ & $\mathrm{B} 1$ & $\mathrm{~B} 1$ \\
\hline Easycook Preta & $75.4 \mathrm{~b}$ & $150.9 \mathrm{e}$ & $75.5 \mathrm{~g}$ & $50.0 \mathrm{~h}$ & $105.2 \mathrm{a}$ & $157.9 \mathrm{a}$ & $29.9 \mathrm{~b}$ & $15.4 \mathrm{~g}$ & $69.8 \mathrm{~b}$ & $3.28 \mathrm{~b}$ & $52.7 \mathrm{~b}$ & $32.9 \mathrm{e}$ & $\mathrm{P}$ & $\mathrm{Br}$ & $\mathrm{B} 1$ & $\mathrm{~B} 1$ \\
\hline Aliança Preta/sel. & $82.2 \mathrm{a}$ & $162.0 \mathrm{c}$ & $79.9 \mathrm{f}$ & $49.3 \mathrm{~h}$ & $102.2 \mathrm{a}$ & $152.7 \mathrm{a}$ & $33.3 \mathrm{a}$ & $12.0 \mathrm{~h}$ & $68.7 \mathrm{~b}$ & $3.84 \mathrm{a}$ & $50.4 \mathrm{~b}$ & $33.0 \mathrm{e}$ & $\mathrm{P}$ & $\mathrm{Br}$ & $\mathrm{B} 1$ & B1 \\
\hline IAC Santa Maria-702 & $82.3 \mathrm{a}$ & $161.6 \mathrm{c}$ & $79.3 \mathrm{f}$ & $49.0 \mathrm{~h}$ & $100.4 \mathrm{a}$ & $142.0 \mathrm{~b}$ & $32.8 \mathrm{a}$ & $10.8 \mathrm{~h}$ & $61.8 \mathrm{~b}$ & $3.78 \mathrm{a}$ & $41.5 \mathrm{c}$ & $29.3 \mathrm{e}$ & $P$ & $\mathrm{Br}$ & $\mathrm{B} 1$ & $\mathrm{~B} 1$ \\
\hline Imperial & $68.5 \mathrm{~d}$ & $141.2 \mathrm{~g}$ & $72.8 \mathrm{~g}$ & $51.5 \mathrm{~h}$ & $55.3 \mathrm{f}$ & $72.0 \mathrm{~h}$ & $22.1 \mathrm{~d}$ & $8.96 \mathrm{i}$ & $57.0 \mathrm{c}$ & $2.43 \mathrm{e}$ & $16.7 \mathrm{f}$ & $23.1 \mathrm{f}$ & $\mathrm{P}$ & $\mathrm{Br}$ & $\mathrm{Br}$ & DB \\
\hline PI- 80.459 & $65.9 \mathrm{e}$ & $142.6 \mathrm{~g}$ & $76.7 \mathrm{f}$ & $53.8 \mathrm{~g}$ & $51.2 \mathrm{~g}$ & $72.2 \mathrm{~h}$ & $22.1 \mathrm{~d}$ & $7.97 \mathrm{i}$ & $53.3 \mathrm{c}$ & $2.51 \mathrm{e}$ & $20.9 \mathrm{e}$ & $29.2 \mathrm{e}$ & $\mathrm{P}$ & $\mathrm{Br}$ & $\mathrm{Br}$ & DB \\
\hline
\end{tabular}

NDF: number of days to the beginning of flowering; NDM: number of days to maturity; RP: reproductive period; \%RP: reproductive period percentage; PHF: plant height at the beginning of flowering; PHM: plant height at maturity; NIM: number of internodes during maturity; HSW: one hundred seed weight; IPY: individual plant yield; AW: agronomic worth; RG: reproductive growth; \%RG: reproductive growth percentage; FC: flower color; PC: pubescence color; CSC: color of seed coat; HC: hilum color; W: white; P: purple; Gy: gray; Y: yellow; Bl: black; Gn: green; L: light; Br: brown; LB: light brown; DB; dark brown.

The first eight soybean genotypes are adapted to Brazilian cultivation conditions; and the last ones are food-type soybean genotypes with small seeds; and the others are food soybean genotypes

Means with diferent letters differ at 1 percent level of significance. 
HSW mean of the 104 genotypes was $29.1 \mathrm{~g}$. The maximum HSW was $42.8 \mathrm{~g} / 100$ seeds for genotypes F82-5767 and F83-8185, which were not different from F82-5722P, F83-8117, F83-8192, and PI-230.977. The minimum HSW was $7.9 \mathrm{~g} / 100$ seeds for PI-80.459, which did not differ from Imperial.

IPY general mean was $60.7 \mathrm{~g} / \mathrm{plant}$. The maximum IPY was $112.3 \mathrm{~g}$ for F82-5782, and was not significantly different from 17 other genotypes. Only two of these were recommended cultivars, industry type, IAC-100 and Davis Marrom, suggesting that there must be food-type soybean genotypes with good adaptability and the possibility of identifying productive genotypes without going through crosses with adapted ones. Food-type soybean adaptation to Brazilian latitude is generally made through crossing with adapted material for gene exchange.

The general mean for AWs was 2.5. The maximum AW was 3.8 for Aliança Preta/sel., which did not differ significantly from IAC-Santa Maria-702, F83-8012, and Araçatuba. The best AWs were for genotypes with average and high IPY, whereas the lowest AW was for genotypes having the lowest IPY and PHM. Those results indicate the presence of positive and significative correlation between AW with IPY and PHM.

The general mean of NDF was 55.5 days. The maximum NDFs was 84.6 days for Doko Preta, for Aliança Preta/sel., and IAC-Santa Maria-702. These three genotypes were later flowering, and had an average RP of 79 days and an average PHF of $100 \mathrm{~cm}$. The minimum NDF rate was 31.2 days for L81-4590. Similar NDFs occurred in for five other genotypes with earlier flowering and maturity with the lower PHF and PHM rates. Great genetic variability for NDF occurred food-type soybean germplasm, in which genes for later flowering in short-day conditions may be present. Under normal cultivation conditions (sowing on November 25) the genotypes which showed higher NDF did not necessarily show better performance on IPY and vice-versa. Destro (1991) detected an average of 34.4 days for NDF in 41 vegetable soybean genotypes with sowing in February. The maximum rate was 41.4 days, indicating they could not be adapted to cultivation either under low latitudes or sown in some other period besides the normal one (February), possibly because they do not have genes for juvenile period. Although variation was lower, the later genotypes had better yield when sown in February.

Toledo et al. (1993) in a genetic analysis of growth in soybean genotypes with determinate grow habit, in three different photoperiod, found that adaptation selection must be performed in each sowing period, either directly selecting plants or the NDF.

NDM general mean was 143.1 days. The maximum NDM was 174.3 days for F83-8175, which was the latest one but was not significantly different from F83-8185 and F85-11346.

RP general mean was 87.6 days. The maximum RP was 112.5 days for F85-11346, which was not significantly different from F81-9136, F82-5722A, and F83-8000. \%RP general mean was $61.4 \%$. The maximum \%RP was $69.7 \%$ for Kanrich and other 16 genotypes.

PHF general mean was $48.2 \mathrm{~cm}$. The maximum PHF was $105.2 \mathrm{~cm}$ for Easycook Preta and for Aliança Preta/sel., IAC-Santa Maria-702, PI-133.226, and Doko Preta, which also had the highest NDF rates and showed positive correlation between NDF and PHF.

PHM general mean was $62.9 \mathrm{~cm}$. The maximum PHM was $157.9 \mathrm{~cm}$ for Easycook Preta, Aliança Preta/sel., Easycook Marrom 4-A, and Easycook Marrom 4, which showed the RG highest rates.

NIM general mean was 16.8 internodes. The maximum NIM was 33.3 internodes for Aliança Preta/sel., being not different from IAC-Santa Maria-702. The highest NIM rates were directly related to the greater rates of PHM and AW.

The general means for $\mathrm{RG}$ and $\% \mathrm{RG}$ were $14.7 \mathrm{~cm}$ and $20.9 \%$, respectively. The maximum rates were $67.6 \mathrm{~cm}$ and $74.5 \%$ for Easycook Preta, not significantly differing from Easycook Marrom 4-A and being considered of indeterminate growth habit. The lowest rate was $0.8 \mathrm{~cm}$ for PI- 80.441 and not differing significantly from 29 other genotypes considered of determinate growth habit.

Aliança Preta/sel. genotype showed the highest NDF, PHF, PHM, NIM, AW and higher rates for 
RG and IPY. Other genotypes with similar performance included Easycook Marrom 4, Easycook Marrom 4-A, Easycook Preta, Aliança Preta/sel., and IAC-Santa Maria-702 . Yellow coating Easycook genotype had the highest \%RP, RG, and \%RG, which means Easycook had earlier flowering with low NDF and the lowest PHF. When the growth was indeterminate it had a better growth after flowering and a higher IPY.

Food-type soybean genotypes exhibit genetic variability among cultivars, which allows the application of breeding programs to obtain adapted cultivars. Genotypes with early flowering under short-day conditions, with short height during flowering, but with a high reproductive period and high reproductive growth, and with undetermined growth habit, may reach a good size and a higher yield.

Field observation has emphasized a higher susceptibility to bedbug in food-type soybean genotypes with a sweet taste. A strategy which could be used to minimize this problem would be selecting later flowering genotypes in short-day conditions, or the incorporation of this characteristic into Asiatic genotypes. This would allow either earlier or later food-type soybean sowing to avoid bedbug infestation. These attacks would be avoided because of the grain-type soybean being sown in November and, when the bedbug population is greater in this culture $\left(R_{3}\right.$ to $R_{7}$ stages of the scale of Fehr \& Caviness (1977)) the food-type soybean will be at other levels, because of the early or late sowing. In the case of late sowing (February) when plants are at levels $\mathrm{R}_{3}$ and $\mathrm{R}_{7}$ (April to June) the temperature will be relatively lower. Consequently, bedbug infestation will be also lower. If the available genetic variability for food-type soybean is used, there is a possibility to control sowing time of cultivars in which bedbug attack is avoided.

\section{CONCLUSIONS}

1. There are some food-type soybean cultivars that are competitive, in terms of yield, with adapted industrial cultivars.

2. Breeding programs oriented to soybean cultivars suitable for direct human consumption are fea- sible through the direct use of Asiatic lines selected to be grown in latitudes which allow a cycle and plant high suitable.

3. Food-type soybean with early flowering in short-day conditions can be easily adapted to Brazilian latitudes if genes for later flowering in shortday conditions are incorporated into them.

\section{ACKNOWLEDGMENTS}

To Conselho Nacional de Desenvolvimento Científico e Tecnológico (CNPq) and Coordenação de Aperfeiçoamento de Pessoal de Nível Superior (CAPES) for grants and financial support.

\section{REFERENCES}

CARRÃO-PANIZZI, M.C. Valor nutritivo da soja e potencial de utilização na dieta brasileira. Londrina: Embrapa-CNPSo, 1988. 13p.

CARRÃO-PANIZZI, M.C.; MEIRA,M.C. Desenvolvimento de cultivares de soja adaptadas para o consumo humano in natura e para a indústria de alimentos. In: EMBRAPA. Centro Nacional de Pesquisa de Soja (Londrina, PR). Resultados de Pesquisa da Soja 1989. Londrina, 1989. p.209-219.

DESTRO, D. Capacidade de combinação de genótipos de soja [Glycine max (L.) Merrill] apropriados para o consumo humano. Piracicaba: ESALQ, 1991, 157p. Tese de Doutorado.

FEHR, W.R.; CAVINESS, C.E. Stages of soybean development. Iowa: Iowa State Univ., 1977. 12p. (Special Report, 80).

HIROMOTO, D.M. Parentais exóticos como fonte de genes para precocidade e produtividade da soja [Glycine max (L.) Merrill]. Piracicaba: ESALQ, 1990. 110p. Tese de Mestrado.

PACOVA, B.E.V. Análise genética de progênies segregantes de soja para o consumo humano. Piracicaba: ESALQ, 1992.217p. Tese de Doutorado.

SANTOS, A.L.C. Variabilidade entre genótipos de soja [Glycine max (L.) Merrill] nas fases imatura e madura de desenvolvimento. Piracicaba: ESALQ, 1988. 135p. Tese de Mestrado. 
SCOTT, A.J.; KNOTT, M.A. A cluster analysis method for grouping means in the analysis of variance. Biometrics, v.30, p.507-512, 1974.

SILVEIRA, I.L.; FLÁVIO, E.F.; OLIVEIRA, S.A.M. Soja: o alimento e a nutrição. Viçosa: UFV, 1989. 58p. (Boletim, 273).

TOLEDO, J.F.F.; OLIVEIRA, M.F.; TSUTIDA, A.C.; KIIHL, R.A.S. Genetic analysis of growth of deter- minate soybean genotypes under three photoperiods. Revista Brasileira de Genética, v.16, p.713-748, 1993.

VELLO, N.A. Ampliação da base genética do germoplasma e melhoramento da soja na ESALQ-USP. In: SIMPÓSIO SOBRE A CULTURA E PRO-DUTIVIDADE DA SOJA. Piracicaba. Anais... Piracicaba: FEALQ, 1992. p.60-81. 